Original Article

\title{
Kinematic, kinetic, and electromyographic characteristics during quiet standing on a balance exercise assist robot
}

\author{
Norihide Itoh, RPT, DMSc ${ }^{1,2)^{*}}$, DAISUke Imoto, RPT ${ }^{2)}$, Shuichi Kubo, RPT ${ }^{3)}$, \\ Masaki Yamaguchi, RPT ${ }^{3)}$, Naoto Shimizu, RPT ${ }^{3)}$, Shogo Toyama, MD, $\mathrm{PhD}^{2}$, \\ Yasuo Mikami, MD, $\mathrm{PhD}^{2,3)}$, Toshikazu Kubo, $\mathrm{MD}, \mathrm{PhD}^{1-3)}$ \\ 1) Department of Advanced Rehabilitation Medicine, Kyoto Prefectural University of Medicine: 465 \\ Kajii-cho, Kawaramachi-Hirokoji, Kamigyo-ku, Kyoto 602-8566, Japan \\ 2) Department of Rehabilitation Medicine, Graduate School of Medical Science, Kyoto Prefectural \\ University of Medicine, Japan \\ 3) Department of Rehabilitation, University Hospital, Kyoto Prefectural University of Medicine, Japan
}

\begin{abstract}
Purpose] The balance exercise assist robot is a training device based on a personal transport assistance robot ridden in the standing position. The personal transport assistance robot uses an inverted pendulum control system and moves in response to movements of the user's center of gravity. The purpose of this study was to describe the characteristics of postural control during the action of stopping the personal transport assistance robot. [Participants and Methods] Eleven healthy male participants were required to maintain a standing position for $30 \mathrm{~s}$; each task was performed 10 times. The measurement conditions were as follows: (1) on the floor; (2) on the robot, touching the handlebars; and (3) on the robot, not touching the handlebars. [Results] During the robotic tasks, the total locus lengths of the center of gravity and total joint momentums of the hip, knee, and ankle joints were larger, and the amount of displacement of the center of pressure was smaller than that during the floor task. Posture control on the robot was performed actively by mechanical interaction of the ankle, knee, and hip joints within a small base of support. [Conclusion] The balance exercise assist robot can be useful for postural control exercises because maintaining a standing position on the personal transport assistance robot required active postural control.

Key words: Postural control, Balance exercise assist robot, Motion analysis
\end{abstract}

(This article was submitted Jun. 5, 2018, and was accepted Jul. 20, 2018)

\section{INTRODUCTION}

In recent years, robotics technology has been applied to the training of patients with gait and balance disorders in clinical rehabilitation settings ${ }^{1)}$, based on the idea that balance training using robotic technology could be useful as a form of postural control training. One of these types of robot, the balance exercise assist robot (BEAR, Toyota Motor Corporation, Toyota, Japan), is based on a personal transport assistance robot (PTAR) ridden in the standing position ${ }^{2}$.

The PTAR is a two-wheeled inverted pendulum system controlled by two motors. The motors are controlled via sensors that detect the posture of the user, to maintain the user in an upright position. Accordingly, when the user moves their center of gravity (COG) forward or backward, the robot also moves forward or backward, respectively. In addition, when the user moves their COG to the left or right, the robot turns in the corresponding direction. In the BEAR system, the PTAR and the computer communicate via Bluetooth, a display cable connects the computer to a large monitor, and the movement of the PTAR is displayed on the monitor as movement of an avatar. Because the user's COG is reflected in the PTAR's movement,

*Corresponding author. Norihide Itoh (E-mail: nitoh@koto.kpu-m.ac.jp)

(C2018 The Society of Physical Therapy Science. Published by IPEC Inc.

(c) (i) $\odot$ This is an open-access article distributed under the terms of the Creative Commons Attribution Non-Commercial No Deriva-

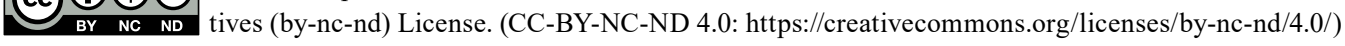


the COG can be visualized, so the device provides useful feedback to the user. Moreover, as the game and robot parameters can be adjusted, balance training can be offered with an optimal degree of difficulty for each individual ${ }^{2}$.

Patients with chronic stroke ${ }^{3)}$ and frail elderly people ${ }^{4}$ showed improvements in dynamic balance ability and lower-limb muscle strength after exercises using BEAR. In healthy participants, a study on physical activity during BEAR exercises described the characteristics of leg muscle activity during three different tasks ${ }^{5}$ ) and the changes in postural strategy during exercise against perturbation ${ }^{6}$. These two previous studies ${ }^{5,6)}$ in healthy participants have focused on movement during the BEAR game task, and did not clarify the user's movement characteristics while operating the PTAR, which involves stopping, forward/backward movement, and left/right turning movement. The purpose of this study was to describe the operation method for stopping the PTAR, that is, the postural control of quiet standing on the PTAR.

\section{PARTICIPANTS AND METHODS}

Eleven healthy male (mean age $26 \pm 5$ years; height $171 \pm 7 \mathrm{~cm}$; weight $62 \pm 7 \mathrm{~kg}$ ) participated in this study. No participant had a history of neurologic or orthopedic disease. All participants provided written informed consent for the experimental procedure, which was approved by the institutional review board of the Kyoto Prefectural University of Medicine (IRB approval number: ERB-C-878). The participants were asked to maintain quiet stance for $30 \mathrm{~s}$ with a distance of $20 \mathrm{~cm}$ between their heels while looking at a marker placed $1.5 \mathrm{~m}$ ahead; the task was performed under three different conditions: (1) on a force plate (GP-5000, Anima Co., Ltd., Tokyo, Japan) on the floor (Floor) (2); on the PTAR touching the handlebars (Handle); and (3) on the PTAR not touching the handlebars (Non-handle). The PTAR (R332 yellow model, Toyota Motor Corporation, Toyota, Japan) employs an inverted pendulum control system, and moves in response to the user's COG movements. Accordingly, when the user moves their COG forward or backward, the PTAR also moves forward or backward. In addition, when the user moves their COG to the left or right, the PTAR rotates. The maximum cruising speed of the PTAR was $2 \mathrm{~m} / \mathrm{s}$. During the Floor and Non-handle tasks, participants stood with their arms crossed against their chests, and in the Handle task, participants were required to rest their hands lightly, but not lean, on the handlebars. Each task was performed 10 times under each of the conditions. The measurement order of the three conditions was chosen at random (Fig. 1).

Marker trajectories were collected at $60 \mathrm{~Hz}$ using a six-camera 3D motion-capture system (KinemaTracer ${ }^{\circledR}$, Kissei Comtec Co., Ltd., Matsumoto, Japan). A total of 16 markers (30 mm in diameter) were placed bilaterally on the acromia, lateral epicondyle of the humerus, ulnar styloid process, iliac crest, hip joint (one-third distance from the great trochanter on a line joining the anterior superior iliac spine and great trochanter), knee joint (midpoint of the anteroposterior diameter of the lateral femoral epicondyle), ankle joint (lateral malleolus), and toes (5th metatarsal head). The COG was calculated based on 14 markers, excluding the iliac crest, taking into account the trunk (including the head and pelvic girdle), the left and right of the upper arm, the forearm, the thigh, the lower leg, and the foot segment. The total locus length and the amount of displacement in the anterior-posterior (AP) and medial-lateral (ML) directions of the COG and the center of pressure (COP) were calculated. Each angle of hip, knee and ankle joint in the sagittal plane was calculated as the motion of the distal segment relative to the proximal segment in the right leg. The mean value, and the amount and total momentum of each joint angle were calculated. The total momentum was defined as the total value of the area formed by joint angles per unit time by trapezoidal approximation.

Force data were measured at $20 \mathrm{~Hz}$ by a force plate during the Floor task, and at $125 \mathrm{~Hz}$ by the PTAR's foot sensors during the Handle and the Non-Handle tasks. The total locus length, and the displacement amount in the AP and ML directions were
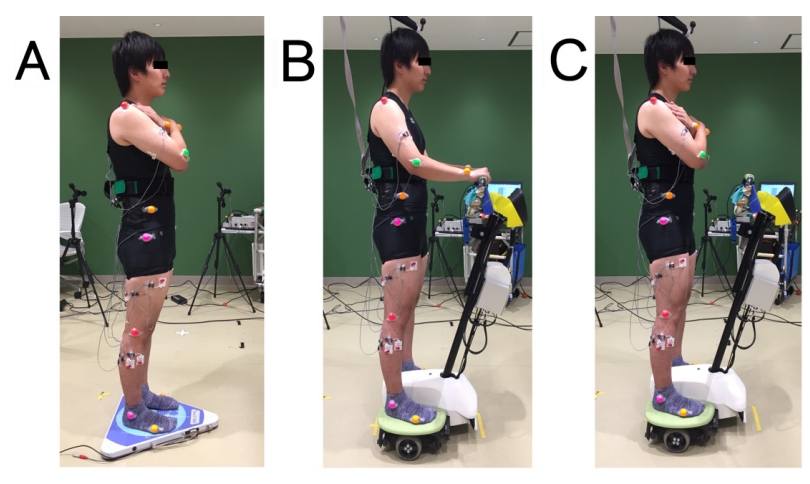

Fig. 1. Experimental conditions. The participant stood on the force plate (A; Floor), or on the robot lightly touching the handlebars (B; Handle), or without touching the handlebars (C; Non-handle); participants' arms were crossed against their chests in A and C conditions; participants were required to rest their hands lightly, but not lean, on the handlebars in the $\mathrm{B}$ condition. A safety harness was used in $\mathrm{B}$ and $\mathrm{C}$ conditions. 
obtained from the COP calculated from the force data stored in the computer after resampling with the KineAnalyzer ${ }^{\circledR}\left(\right.$ Kissei $^{\circ}$ Comtec Co., Ltd., Matsumoto, Japan).

Surface electromyography (EMG) data were collected using a wireless EMG device (MQ16, Kissei Comtec Co., Ltd., Matsumoto, Japan). Muscle activity was recorded for each of the following six muscles in the right lower leg: gluteus medius (GM); rectus femoris (RF); biceps femoris (BF); tibialis anterior (TA); medial head of the gastrocnemius (MG); and peroneus longus (PL). The electrodes were placed on the skin according to SENIAM conventions ${ }^{7}$. The EMG data were acquired at a sampling frequency of $1,000 \mathrm{~Hz}$, and the raw EMG signals were bandpass filtered between 20 and $500 \mathrm{~Hz}$ using the KinemaTracer ${ }^{\circledR}$ to remove low frequency motion artifact and high frequency noise. These signals were then processed for rectification and integrated every $100 \mathrm{~ms}$. For each participant, EMGs were normalized to the corresponding maximum voluntary contraction (MVC) to reduce intersubject variability.

A mean value was calculated for each study parameter from the 110 repeats of the quiet stance task performed under the three experimental conditions.

In order to synchronize the data collection, the force plate and the EMG device were triggered by a start signal from the 3-D motion analysis system in the Floor task. The 3-D motion analysis system, PTAR, and EMG device were triggered by a start signal from a custom-made trigger box in the Handle and Non-handle tasks.

A repeated measures analysis using a mixed model was used to identify statistically significant differences in kinetic and kinematic values and lower-leg muscle activities for the three conditions. In this study, the fixed effects were the three conditions, and these models also estimated random effects due to differences in participants. In the analysis, $p$ values $<0.05$ were considered statistically significant. For the post-hoc test, the Bonferroni correction for multiple comparisons was used, and $\mathrm{p}$ values $<0.0167$ were considered statistically significant since three pair-wise comparisons were done on each data set (i.e., $\mathrm{p}<0.05 / 3=\mathrm{p}<0.0167$ ). JMP ${ }^{\circledR}$ Pro 12 (SAS Institute Inc., Cary, NC, USA) was used for statistical analyses.

\section{RESULTS}

Table 1 shows results for the measured parameters under Floor, Handle, and Non-handle conditions.

The largest total locus length of COG and largest amount of displacement of COG in the AP direction were observed in the Non-handle task, followed by the Handle and Floor tasks, and the smallest amount of displacement of COP in the AP direction was observed in the Non-handle task, followed by the Handle and Floor tasks. The total locus length of COP in the Non-handle task was larger than in the Floor and Handle tasks. The amount of displacement of COG in the ML direction was smaller in the Handle task than in the Floor and Non-handle tasks, and the amount of displacement of COP in the ML direction was smaller in the Handle and Non-handle tasks than in the Floor task.

The mean of hip joint angle in the Non-handle task was the highest flexion, followed by the Handle and then Floor tasks. The mean of knee joint angle was higher flexion in the Handle and Non-handle tasks than the Floor task. The mean of ankle joint angle under all conditions was dorsiflexion, with no significant difference between the three conditions. The angular displacement of all lower leg joints was larger in the Non-handle than the Floor task; in addition, the ankle angular displacement was larger in the Handle task than in the Floor task. The total momentum of all the lower leg joints increased in the Handle and Non-handle tasks compared with the Floor task; in addition, the total momentum of the hip joint increased in the Non-handle task compared with the Handle task.

The highest mean muscle activities of the GM, RF, and TA were observed in the Non-handle task, followed by the Handle and then the Floor tasks. The mean muscle activity of BF was lower for the Handle task than for the Floor task, and the MG activity was lower in the Handle and Non-handle tasks than in the Floor task. Finally, mean muscle activity of the PL was higher in the Non-Handle task than in the Floor and Handle tasks.

\section{DISCUSSION}

With or without handlebar use, the results for COG and COP showed that the standing positions on the PTAR were an unstable environment, and the results for joint motion and muscle activity of the lower limbs showed that quiet standing on the PTAR required different postural control to standing on the floor. In addition, standing on the PTAR without touching the handlebars was a less stable environment, and increased joint movement and muscle activity of the lower limb were observed.

To stop the PTAR, the user's COG must be matched to the center of the PTAR body. Although the participants tried to reduce the amount of COP displacement and make their COG match, it was difficult to perfectly coincide the two and, since the position of the COG was reflected in the movement of the PTAR, the total length and the amount of displacement in the AP direction of the COG increased. The PTAR turns when load symmetry is lost. When standing on the PTAR, the participants' consciousness of the bilateral symmetry of the load was greater and the amount of displacement of COP in the ML direction was smaller than when standing on the floor.

The standing posture on the PTAR was flexion of the lower limb joints, the range of motion of the ankle joint was large, and the total momentum of all lower leg joints was large compared with the Floor condition. Hsu et al. reported that displacement of six angles in the longitudinal direction - the ankle, knee, hip, lumbosacral, 7th cervical-1st thoracic, and atlanto-occipital joints - was coordinated to minimize the impact on the center ${ }^{8}$. In this study, posture was also controlled 
Table 1. Comparison of measured parameters during Floor, Handle, and Non-handle conditions

\begin{tabular}{|c|c|c|c|}
\hline & Floor & Handle & Non-handle \\
\hline \multicolumn{4}{|l|}{$\operatorname{COG}(\mathrm{cm})$} \\
\hline Total locus length & $22.6(17.5)$ & $34.8(19.5)^{*}$ & $41.6(16.6)^{*}, * *$ \\
\hline Amount of displacement in AP direction & $1.9(1.1)$ & $7.9(3.8)^{*}$ & $11.4(6.2)^{*}, * *$ \\
\hline Amount of displacement in MP direction & $0.9(0.5)$ & $0.7(0.4)^{*}$ & $0.9(0.7)^{* *}$ \\
\hline \multicolumn{4}{|l|}{$\mathrm{COP}(\mathrm{cm})$} \\
\hline Total locus length & $26.2(10.3)$ & $23.2(16.0)$ & $36.3(14.5)^{*}, * *$ \\
\hline Amount of displacement in AP direction & $2.5(1.6)$ & $1.9(1.7)^{*}$ & $1.3(0.6)^{*}, * *$ \\
\hline Amount of displacement in MP direction & $1.2(0.7)$ & $0.2(0.2)^{*}$ & $0.3(0.2)^{*}$ \\
\hline \multicolumn{4}{|l|}{ Joint angle } \\
\hline \multicolumn{4}{|c|}{ Mean value (degrees, +: flexion/dorsiflexion, -: extension/plantarflexion) } \\
\hline Hip & $-0.3(2.9)$ & $2.2(4.0)^{*}$ & $5.4(4.7) * * *$ \\
\hline Knee & $1.2(2.2)$ & $3.5(4.0)^{*}$ & $4.0(4.0)^{*}$ \\
\hline Ankle & $3.0(2.0)$ & $2.6(3.6)$ & $3.3(3.0)$ \\
\hline \multicolumn{4}{|l|}{ Amount of displacement (degrees) } \\
\hline Hip & $2.6(1.6)$ & $2.9(1.4)$ & $3.2(1.6)^{*}$ \\
\hline Knee & $1.8(1.9)$ & $2.3(1.9)$ & $2.5(2.1)^{*}$ \\
\hline Ankle & $1.9(1.3)$ & $2.7(1.0)^{*}$ & $2.7(1.1)^{*}$ \\
\hline \multicolumn{4}{|l|}{ Total momentum (degrees $\times$ sec) } \\
\hline Hip & $153.3(105.1)$ & $229.8(141.5)^{*}$ & $323.7(168.4)^{*}, * *$ \\
\hline Knee & $56.0(48.4)$ & $123.6(97.8)^{*}$ & $131.8(107.4)^{*}$ \\
\hline Ankle & $90.1(58.9)$ & $115.6(72.0)^{*}$ & $114.3(66.0)^{*}$ \\
\hline \multicolumn{4}{|l|}{ Average muscle activity (\%MVC) } \\
\hline Gluteus medius & $3.6(1.5)$ & $4.3(1.7)^{*}$ & $5.9(2.8)^{*}, * *$ \\
\hline Rectus femoris & $2.6(1.9)$ & $5.0(3.9)^{*}$ & $7.0(5.0)^{*}, * *$ \\
\hline Biceps femoris & $4.2(4.4)$ & $3.0(2.4)^{*}$ & $3.6(2.8)$ \\
\hline Tibialis anterior & $1.8(1.1)$ & $3.8(3.1)^{*}$ & $7.2(3.7)^{*}, * *$ \\
\hline Medial head of gastrocnemius & $6.0(4.4)$ & $3.2(3.4)^{*}$ & $3.7(2.9)^{*}$ \\
\hline Peroneus longus & $5.3(4.8)$ & $4.4(3.9)$ & $7.5(7.0)^{*}, * *$ \\
\hline
\end{tabular}

Values are displayed as mean (standard deviation).

*indicates significantly different to Floor condition $(\mathrm{p}<0.0167)$. **indicates significantly different to Handle condition $(\mathrm{p}<0.0167)$.

through the mechanical interaction of the ankle, knee, and hip when maintaining a standing position on the PTAR. The amount of displacement at the ankle has been reported to increase when standing still on unstable seesaws ${ }^{9}$. On the unstable seesaws, the participants were able to keep balance mainly by moving the ankle joint, whereas the hip and knee movements were within the range seen when the individuals stood still on a rigid platform ${ }^{9}$. The amount of displacement at the ankle joint was also greater on the unstable PTAR in this study than when standing on the floor, similar to the results observed with seesaws. To achieve smooth control of standing on the PTAR, ample joint freedom had to be coordinated chronologically and spatially; the posture of bending the leg joint might, therefore, have been selected.

Compared with the Floor condition, lower-limb muscle activity under the Handle condition was higher for the RF and TA and smaller for the BF and MG. The activity of the ventral lower limb muscles becomes greater when the COG is moved backward $^{10}$. During the Handle condition, the standing posture was flexion of the hip and knee joints, and it appears that the COG was displaced rearward of the COP, compared with the Floor condition. Also, the demand for lateral control of the COG was high under PTAR conditions, so the muscle activity of the GM increased. ML balance is under hip (abduction/adduction) control in side-by-side stance ${ }^{11)}$. This agrees with our results.

The study also examined the influence of the handlebars on the PTAR. In the Non-handle task, the total locus length of the COG and COP, and the COG displacement amount in the AP/ML directions were larger and more unstable than during the Handle task. The COP displacement amount was small in the AP direction, and there was no between-group difference for the ML direction. It is well known that a light and voluntary touch with a fingertip on a fixed surface improves postural stability during quiet standing. It was thought that tactile feedback provided by fingertip contact contributes to the reduction of postural sway ${ }^{12}$. Thus, the light contact with the handlebars in the current study may have contributed to postural stability on the PTAR. The postural strategies for quiet standing include the ankle strategy and hip joint strategy. The ankle 
strategy persists during small perturbations consisting of low-amplitude, low-velocity, or low-frequency stimuli. With larger perturbations, the hip strategy predominated ${ }^{10)}$. In the Non-handle task, it is assumed that muscle activity in the GM and RF was large because the total locus length and displacement of the COG are large, to make heavy use of the hip strategy. In addition, because the demand for COG control in the ML direction increased in the Non-handle task, muscle activity in the GM and PL was high.

Some limitations of the present study must be acknowledged when interpreting the results. In this study, head, neck, and trunk motion were not analyzed. Furthermore, the influence of both upper limbs has not been sufficiently studied. The influence of both upper limbs on postural control was small because participants' arms were crossed against their chests in the Floor and Non-handle conditions. On the other hand, in the Handle condition, the participants were instructed not to lean on the handlebars. However, the amount of load on the handlebars and the motion of the hands were not measured. The possibility that contact with the handlebars affected the control of the PTAR cannot be excluded.

In conclusion, standing on the PTAR required performance of more active postural control under these unstable conditions than standing on the floor. Therefore, the BEAR may be useful for providing postural control exercises. In addition, since the handlebars make it easier to achieve postural control, the BEAR can be used for patients with severe balance disorders.

\section{Funding}

This research did not receive any specific grant from funding agencies in the public, commercial, or not-for-profit sectors.

\section{Conflict of interest}

Toyota Motor Corporation supported the study through the loan of a balance exercise assist robot.

\section{REFERENCES}

1) Shirota C, van Asseldonk E, Matjačić Z, et al.: Robot-supported assessment of balance in standing and walking. J Neuroeng Rehabil, 2017, 14: 80. [Medline] [CrossRef]

2) Ozaki K, Kagaya H, Hirano S, et al.: Preliminary trial of postural strategy training using a personal transport assistance robot for patients with central nervous system disorder. Arch Phys Med Rehabil, 2013, 94: 59-66. [Medline] [CrossRef]

3) Tsunoda T, Hirano S, Saitoh E, et al.: The game task level of a balance exercise assist robot achieved by chronic stroke patients with hemiplegia is correlated with balance ability after training. Jpn J Compr Rehabil Sci, 2016, 7: 87-94.

4) Ozaki K, Kondo I, Hirano S, et al.: Training with a balance exercise assist robot is more effective than conventional training for frail older adults. Geriatr Gerontol Int, 2017, 17: 1982-1990. [Medline] [CrossRef]

5) Ishihara K, Hirano S, Saitoh E, et al.: Characteristics of leg muscle activity in three different tasks using the balance exercise assist robot. Jpn J Compr Rehabil Sci, 2015, 6: 105-112.

6) Itoh N, Tanabe S, Hirano S, et al.: Changes in postural strategy during exercise against perturbation using the balance exercise assist robot: a pilot study. J Phys Ther Sci, 2017, 29: 16-19. [Medline] [CrossRef]

7) Hermens HJ, Freriks B, Disselhorst-Klug C, et al.: Development of recommendations for SEMG sensors and sensor placement procedures. J Electromyogr Kinesiol, 2000, 10: 361-374. [Medline] [CrossRef]

8) Hsu WL, Scholz JP, Schöner G, et al.: Control and estimation of posture during quiet stance depends on multijoint coordination. J Neurophysiol, 2007, 97: 3024-3035. [Medline] [CrossRef]

9) Almeida GL, Carvalho RL, Talis VL: Postural strategy to keep balance on the seesaw. Gait Posture, 2006, 23: 17-21. [Medline] [CrossRef]

10) Horak FB, Nashner LM: Central programming of postural movements: adaptation to altered support-surface configurations. J Neurophysiol, 1986, 55: 13691381. [Medline] [CrossRef]

11) Winter DA, Prince F, Frank JS, et al.: Unified theory regarding A/P and M/L balance in quiet stance. J Neurophysiol, 1996, 75: 2334-2343. [Medline] [CrossRef]

12) Kouzaki M, Masani K: Reduced postural sway during quiet standing by light touch is due to finger tactile feedback but not mechanical support. Exp Brain Res, 2008, 188: 153-158. [Medline] [CrossRef] 